\title{
Novel Mutation in LARP7 in Two Iranian Consanguineous Families with Syndromic Intellectual Disability and Facial Dysmorphism
}

\author{
Goli Kazemi, MSc ${ }^{1}$; Fatemeh Peymani, MSc ${ }^{1}$; Marzieh Mohseni, MSc ${ }^{1,2}$; Farzane Zare Ashrafi, MSc ${ }^{1}$; Sanaz Arzhangi, MSc ${ }^{1}$; Fariba Ardalani, \\ MSc'; Fatemeh Aghakhani Moghaddam, BSc ${ }^{1}$; Kimia Kahrizi, MD ${ }^{1}$; Hossein Najmabadi, PhD ${ }^{1 *}$
}

${ }^{1}$ Genetics Research Center, University of Social Welfare and Rehabilitation Sciences, Tehran, Iran

${ }^{2}$ Student Research Committee, University of Social Welfare and Rehabilitation Sciences, Tehran, Iran

\begin{abstract}
Background: Recently, we have reported mutations in LARP7 gene, leading to neurodevelopmental disorders (NDDs), the most frequent cause of disability in children with a broad phenotype spectrum and diverse genetic landscape.

Methods: Here, we present two Iranian patients from consanguineous families with syndromic intellectual disability, facial dysmorphism, and short stature.

Results: Whole-exome sequencing (WES) revealed a novel homozygous stop-gain (c.C925T, p.R309X) variant and a previously known homozygous acceptor splice-site (c.1669-1_1671del) variant in LARP7 gene, indicating the diagnosis of Alazami syndrome. Conclusion: These identified variants in patients with Alazami syndrome were consistent with previously reported loss of function variants in LARP7 and provide further evidence that loss of function of LARP7 is the disease mechanism.

Keywords: Intellectual disability, LARP7, Mutation, Phenotype, Whole exome sequencing

Cite this article as: Kazemi G, Peymani F, Mohseni M, Zare Ashrafi F, Arzhangi S, Ardalani F, et al.. Novel mutation in LARP7 in two Iranian consanguineous families with syndromic intellectual disability and facial dysmorphism. Arch Iran Med. 2020;23(12):842847. doi: 10.34172/aim.2020.112.
\end{abstract}

Received: June 1, 2020, Accepted: Setember 20, 2020, ePublished: December 1, 2020

\section{Introduction}

Neurodevelopmental disorders (NDDs), the most frequent cause of disability in children with complex genotypephenotype correlations, are the main reasons for most referrals to health services. Alazami syndrome (ALAZS; MIM 615071) is a rare syndromic developmental disorder characterized by short stature, and distinct facial and skeletal features, with the major hallmark of neurodevelopmental delay and intellectual disabilities. ${ }^{1}$ This syndrome is caused by biallelic homozygous or compound heterozygous mutations in LARP7 (La Ribonucleoprotein domain family member 7), located on chromosome $4 \mathrm{q} 25$, that was originally identified by Najmabadi et al, in 2011. ${ }^{1,2}$ LARP7 mutations were identified in 2008 by $\mathrm{He}$ et al, ${ }^{3}$ and in 2011 imputed as a new causative gene involved in autosomal recessive intellectual disability by Najmabadi et al. ${ }^{1,4}$

$L A R P 7$ acts as a negative regulator of polymerase II genes, the crucial parts of transcription/translation regulation. Functional studies have shown that $L A R P 7$ is ubiquitously expressed in all tissues with an exceptional increase in the brain. ${ }^{2,3}$ The LARP7 protein with 582 amino acids (aa) acts as a stabilizing chaperone molecule of an abundant non-coding RNA (ncRNA) 7SK, ${ }^{2,4}$ negatively impacting the expression of a wide variety of other genes through its inhibitory effect on positive transcription elongation factor b (P-TEFb), as well as competing with HMGA1mediated transcriptional regulation..$^{5-7}$

LARP7, MEPCE, and HEXIM1/2 proteins, whose functions are critical in neuronal development, and 7SK non-coding RNA compose 7SK snRNP which plays important roles in regulating both RNAPII-mediated mRNA elongation and small nuclear/nucleolar RNA synthesis. ${ }^{8,9}$

Although it was assumed that LARP7 deficiency enhances cell proliferation due to increasing the cellular activity of P-TEFb, it has been indicated that Larp7 knockdown in embryonic stem cells (ESCs) is associated with growth failure. Larp7 deficiency stimulates ESCs differentiation instead of cell divisions via down regulation of Lin28, a positive regulator of organismal growth. This effect of Larp7 deficiency could explain the molecular etiology of primordial dwarfism seen in patients with loss of function mutations in LARP7..10,11

Here, we present clinical and molecular findings in two Iranian patients from consanguineous families with homozygous mutations in LARP7 gene. 


\section{Materials and Methods}

Clinical Report

Two patients were referred to the Genetics Research Center (GRC), at the University of Social Welfare and Rehabilitation Sciences (USWR), Tehran, Iran, as rare and undiagnosed cases for further molecular investigation.

Patient I was a 15-year-old male born to healthy, consanguineous parents. $\mathrm{He}$ was born after an unremarkable pregnancy with $\mathrm{C} / \mathrm{S}$ delivery and neonatal period. His birth weight was $3120 \mathrm{~g}$, height was $49 \mathrm{~cm}$, and occipitofrontal head circumference (OFC) was $34 \mathrm{~cm}$. His early development was normal and he started sitting at 8 months and standing at 10 months but later developed psychomotor delay. He had delayed speech development and was noted to have progressive spasticity and hypertonia during infancy and he could not walk without assistance. He had a history of seizure, which started at 2 years of age and was controlled with medication. At 4 years, he had a heart operation to correct a congenital atrial septal defect. On examination at 15 years, his height was $128 \mathrm{~cm}(-5.4$ SD), his weight was $17 \mathrm{~kg}(-3.3 \mathrm{SD})$ and $\mathrm{OFC}$ was $48 \mathrm{~cm}$ (-4.6 SD). He had short stature, microcephaly, thin body and ataxic gait. He had facial dysmorphism including a broad forehead, wide nasal bridge, broad nose, malar hypoplasia, retrognathia, full lips and low set protruding ears. He had spasticity, hyperreflexia and scoliosis. Brain MRI at the age of 15 years demonstrated an increased signal in periventricular white matter while EEG did not show any abnormal epileptic waves. Cognitive status, evaluated using WAIS-IV, showed an IQ of 30, in the range of severe intellectual disability (ID) (Figure 1).

Patient II was a 25-year-old woman, the second child of healthy consanguineous parents. She was born at term after an uneventful pregnancy with normal vaginal delivery. She had normal birth OFC and height, but a low birth weight that had not been documented. She revealed severe early psychomotor developmental delay and started sitting, standing and walking at the age of 24 months. She had delayed speech development and her first words were at 3 years. She had no history of seizure. She developed a bilateral cataract at the age of 25 years. On examination, at 25 years, her height was $140 \mathrm{~cm}(-3.6$ SD), her weight was $30 \mathrm{~kg}(-4.1 \mathrm{SD})$, and OFC was 53 $\mathrm{cm}(-0.5 \mathrm{SD})$. She had proportional short stature and thin body. She had facial dysmorphism, including a broad forehead, wide nasal bridge, broad nose, malar hypoplasia, short philtrum, prognathism, full lips and low set ears. She walked normally with no ataxic or spastic gait and her extremities were normal. Cognitive status, evaluated using WAIS-IV, showed an IQ of 40, in the range of severe ID; she had stereotypic movement and speech, anxiety and self-injurious behavior. In laboratory testing, she showed hypercholesterolemia, normal cortisol and decreased adrenocorticotrophic hormone level (Figure 2).

The study was approved by the Ethics Committee of the USWR and written informed consent was obtained from the parents of the two patients for further molecular analysis and to take photographs of the patients. After obtaining an informed consent, complete clinical examination and genetic counseling were performed.

\section{Whole-Exome Sequencing}

A 10-mL sample of whole blood was collected from each patient using EDTA as anticoagulant. Genomic DNA was extracted, using the salting-out method, and the integrity and quantity of the extracted DNA were determined. Exome capture of the two proband's DNA was accomplished using the Agilent SureSelectXT2 kit (Version 6) (Agilent Technologies, Inc., Santa Clara, CA), followed by paired-end sequencing on Illumina HiSeq2500's flowcell (Illumina Inc., San Diego, CA). Mapping FASTQ files to the reference human genome (human genome 19 version, Genome Reference Consortium GRCh37) was performed by applying the Burrows-Wheeler transform algorithm. ${ }^{12}$ The Picard toolkit and GATK package were used for trimming, filtering, base recalibration, coverage determination and insertion/deletion realignment of the SAM files, and then variants were called using the Unified
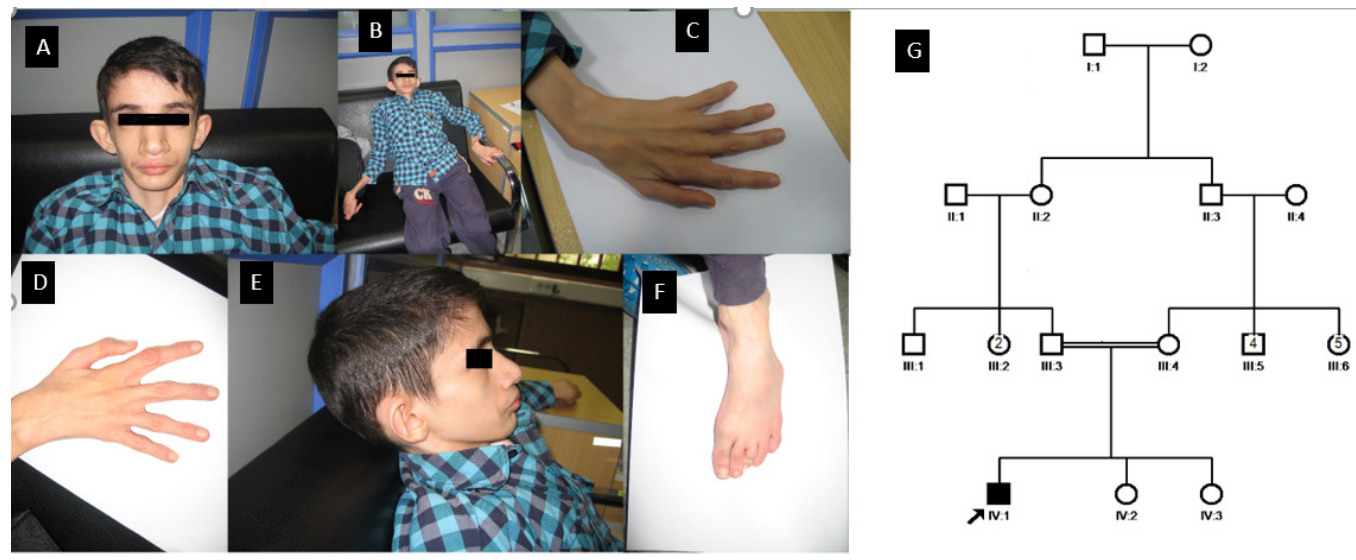

Figure 1. Symptoms of Patient I. A) Facial features (wide nasal bridge, broad nose, retrognathia, full lips and low set protruding ears). B) Motor developmental delay, skeletal problems. C and D) Skeletal problems. E) Broad forehead. F) Skeletal problems. G) Pedigree of the family. 


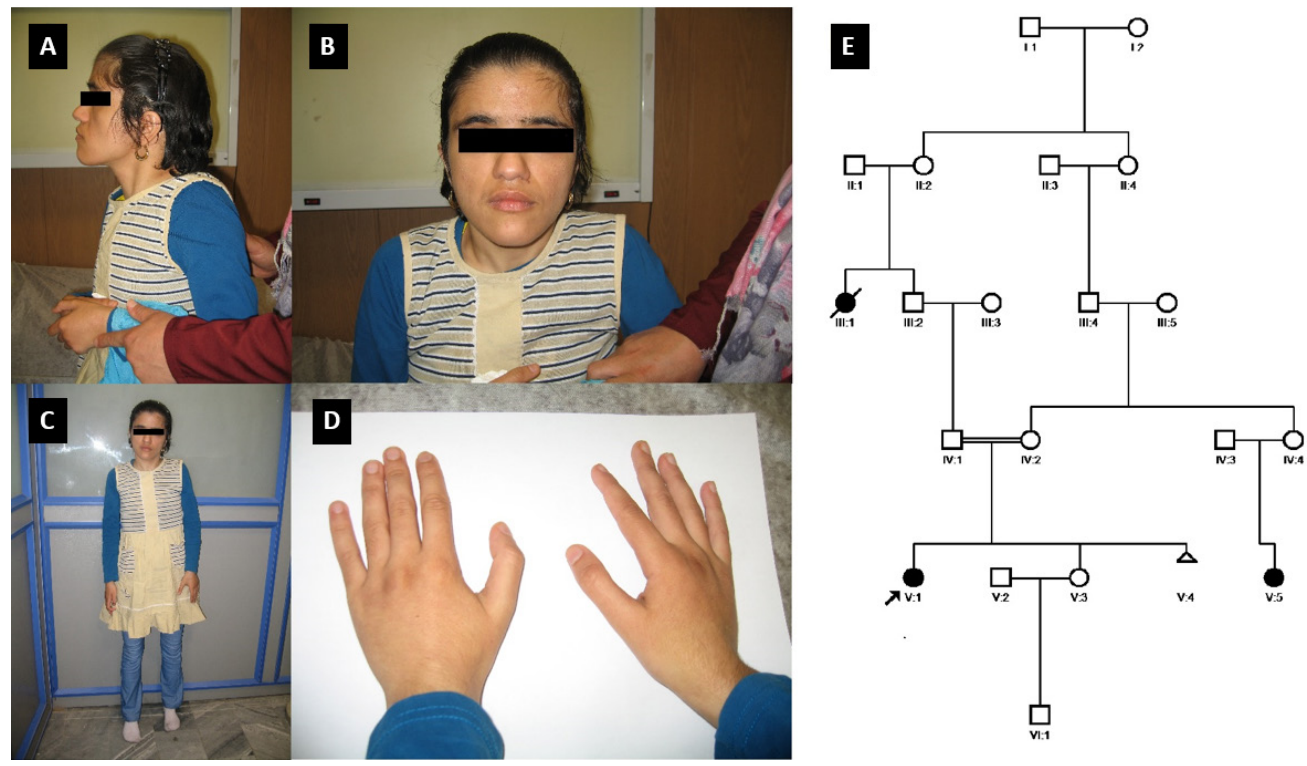

Figure 2. Symptoms of Patient II. A and B) Facial features of the proband (broad forehead, wide nasal bridge, broad nose, malar hypoplasia, short philtrum, prognathism, full lips and low set ears). C) Short status. D) Skeletal problems (hand deformity). E) Pedigree of the family.

Genotyper module of the GATK package. ${ }^{13-15}$ ANNOVAR tool was then used to annotate called variants. ${ }^{16}$ Variants with global minor allele frequency (MAF) $\leq 0.01$ were considered for more filtration using available databases including 1000 Genome Project, ESP6500, Genome Aggregation Database (gnomAD), Exome Aggregation Consortium (ExAC), Kaviar, and ABraOM database. ${ }^{17-21}$ Considering the consanguineous background of the two families, all synonymous, intronic, and heterozygous variants, were filtered out. The variants that have been presented in homozygous status in gnomAD and Iranome (Iranian normal control database, http://www.iranome. $\mathrm{com} /)^{22}$ and predominantly predicted to be benign, using bioinformatics tools including SIFT, Mutation Taster, FATHMM MKL, Mutation Assessor, Polyphen 2, and CADD, were filtered out. Genotype-phenotype correlation, VarSome, and American College of Medical Genetics and Genomics (ACMG) guidelines were used for the identification of causal variants which were further validated by conventional Sanger sequencing and cosegregation analysis in the available family members. ${ }^{23,24}$ In addition, other bioinformatics tools, such as NetGene2 Server and Human splicing finder 3.1, were utilized to assess the consequence of splice-site mutations in silico. ${ }^{25,26}$ InterPro (https://www.proteomicsdb.org) and STRING databases (string-db.org) were respectively used to evaluate the putative structural changes and functional characterizations of the LARP7 protein. ${ }^{27,28}$

\section{Results}

Diagnosis by Whole-Exome Sequencing

Whole-exome sequencing (WES) of patients in the studied families (8900195 and 96000005) revealed two homozygous pathogenic variants in LARP7 (NM_016648). A novel stop-gain pathogenic variant defined as chr4:113568633C>T, c.C925T (p.Arg309X) in exon 7 of LARP7 in patient I of family 8900195, and a previously known pathogenic deletion variant, affecting the splice acceptor site, defined as chr4:113578398 TTTAG>T, c.1669-1_1671del in intron 12 of LARP7 in patient II of family 96000005 , were identified.

Sanger sequencing of the candidate variants in patients and their parents confirmed the homozygous status of the variants in the patients and the heterozygous status of the variants in their parents (Figures 3 and 4).

A schematic representation of the protein domains of Larelated protein 7 , encoded by LARP7, with the positions of the two variants identified is shown in Figure 5.

\section{Discussion}

The novel homozygous variant identified in patient I (c.C925T, p.R309X) was a transition substitution of $\mathrm{T}$ to $\mathrm{C}$, residing at exon 7 of LARP7. This variant causes a premature termination codon (PTC) leading to the C-terminus truncation of 273 amino acids remaining with 309 amino acids, which is roughly about a half-length of the normal protein $(582$ aa). The aforementioned mutation at Arg309 would cause significant structural loss at the C-terminal end of the protein, including RNA Recognition Motif 2 (RRM2), which is needed for specific binding to 7SK and P-TEFb (Figure 5). Nevertheless, the presence of the PTC in the transcript probably causes early mRNA degradation by nonsense-mediated RNA decay (NMD), in order to prevent aberrant protein synthesis.

In patient II, a 4-bp intronic deletion (c.16691_1671del) was found, which affects the acceptor splicing 


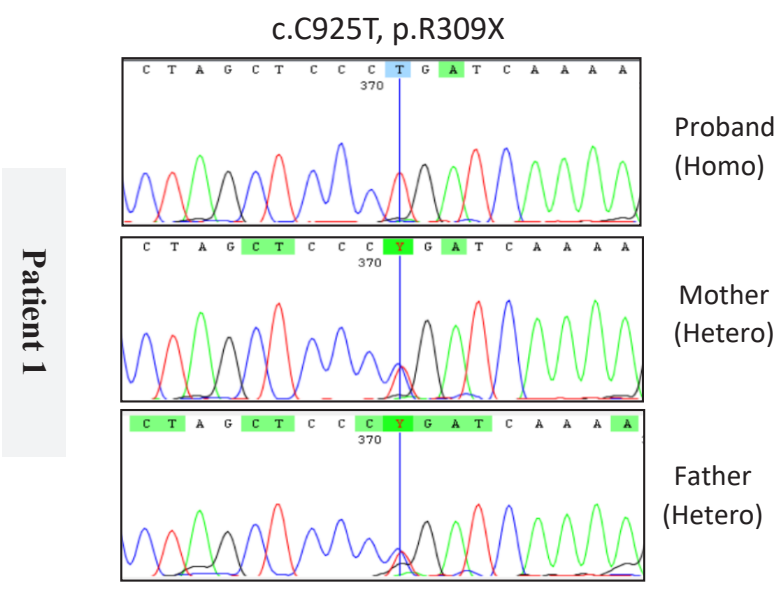

Figure 3. DNA Sequence Chromatograms Showing Variant (c.C925T p.R309X) in Heterozygous Status in Normal Individuals (Mother and Father), and in Homozygous State in the Affected Individual (Patient I) of Family 8900195

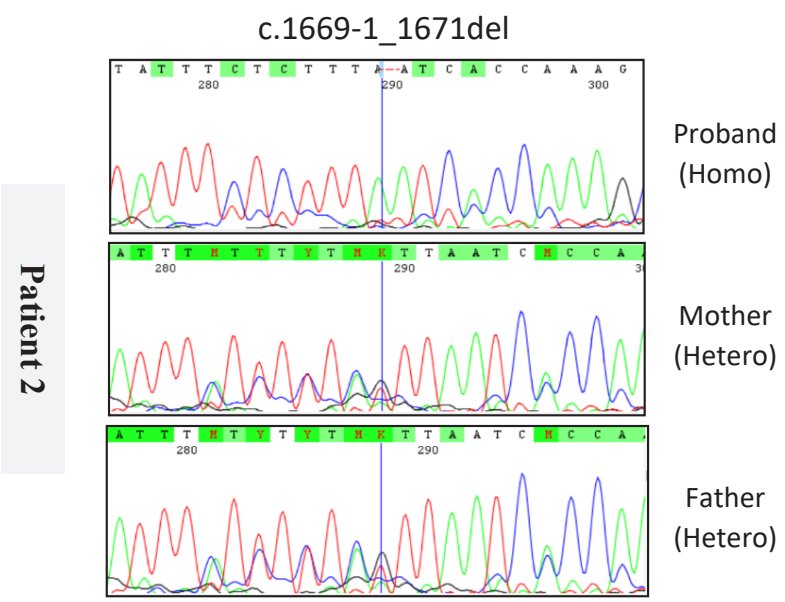

Figure 4. DNA Sequence Chromatograms Showing Variant (c.16691_1671del) in Heterozygous State in Normal Individuals (Mother and Father) and in Homozygous State in the Affected Individual (Patient II) of Family 96000005

site adjacent to exon 13. In this patient, the distinct AG dinucleotide at the acceptor splice-site of intron 12 was deleted; therefore, this intron could not be spliced out properly. In silico prediction tools (such as NetGen2 and Human Splicing Finder) predicted the potential pathogenic effect of the c.1669-1_1671del variant on splicing through a loss of the acceptor splicing site.

The stop-gain variants and deletions variants affecting the splice-sites, two variants reported in this study, would result in truncated proteins. Such protein-truncating variants (PTVs) are usually subject to nonsense-mediated RNA decay (NMD), which prevents production of truncated proteins, leading to complete loss of function of that copy of the protein. ${ }^{29}$ Based on an extensive literature review, we could make the assumption that our identified pathogenic PTVs are predicted to trigger the NMD process and the consequent loss of function of LARP7. ${ }^{22-30}$ As the presence and stable attachment of LARP7 to the 7SK small nuclear RNA are crucial for the function of the 7SK snRNP complex, ${ }^{6,31,32}$ it could be concluded that a nonfunctional 7SK snRNP complex due to the mentioned loss of function effects of the variants, is responsible for the clinical manifestations observed in our patients.

The clinical features of Alazami syndrome were first described in 2012 by Alazami et al in a study of nine patients from a large multiplex Saudi family. All patients in that study presented with short stature, distinctive facial features, motor developmental delay and intellectual disabilities. ${ }^{2}$ More typical phenotypic features of Alazami syndrome have been reported more recently, including more distinctive facial features such as triangular face, prominent forehead, deep-set eyes, sparse eyebrows, broad nose, widely spaced teeth and wide mouth, and also some behavioral problems such as anxiety and hypersensitivity to auditory stimuli. ${ }^{7,31}$ Ivanovski et al have recently provided a comprehensive review of the most common features of Alazami syndrome, along with a case report with childhood thyroid carcinoma besides typical features of Alazami syndrome. ${ }^{33}$ Regarding the phenotype expansion, our patients presented some less common symptoms including heart abnormalities (moderate mitral regurgitation), and intractable seizure and presented bilateral cataract as a new symptom.

To date, 24 patients from 12 families have been reported to be affected. ${ }^{7,31,33-35}$ After Alazami et al, Najmabadi et al have reported the highest number of patients with mutation in LARP7, including 6 patients from four Iranian families. ${ }^{1,2,4,36,37}$ Altogether, the four Alazami cases

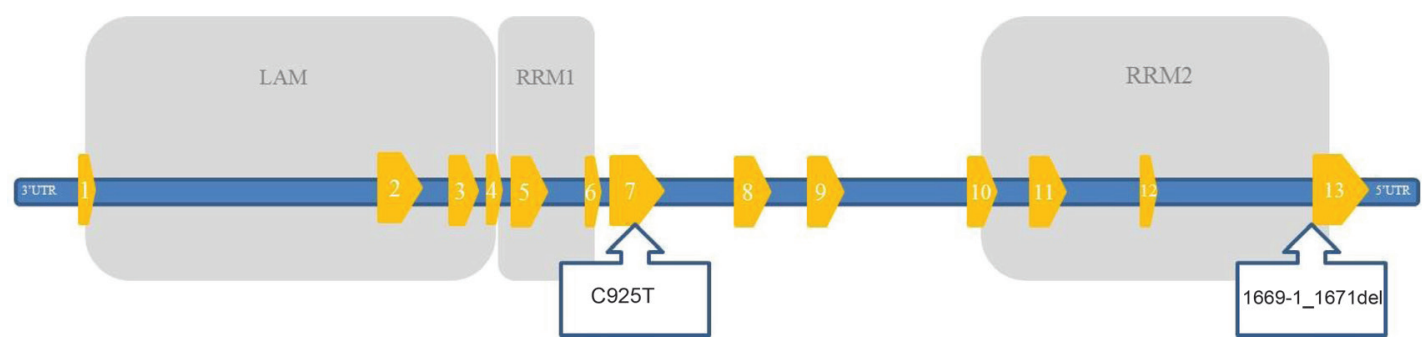

Figure 5. Schematic Representation of the Protein Domains of La-Related Protein 7, Encoded by LARP7, with Positions of the Two Identified Variants, Including a Novel Stop-Gain (c.C925T, p.R309X) and an Acceptor Splice-Site (c.1669-1_1671del) Variant (LAM, Lupus Antigen Motif; RRM1, RNA Recognition Motif 1; RRM2, RNA Recognition Motif 2). 
previously reported by our team, and the two patients reported here, are from consanguineous families. ${ }^{38}$

Furthermore, all the previously reported LARP7 variants are loss of function variants including mainly frameshift variants, donor splice site variants, intronic variants and nonsense variants. ${ }^{1,2,4,7,31,33-37,39}$ Here, we present two other pathogenic loss of function variants, one novel stop-gain variant and one acceptor splice-site variant, which testify to the notion that complete loss of function of LARP7 is likely the disease mechanism.

The present study described the clinical phenotypes and molecular findings in two Iranian patients with Alazami syndrome, expanding the genotypic and phenotypic spectrum of this syndrome. Next generation sequencing in all its different forms has become a powerful tool in solving cases with rare genetic disorders, including undiagnosed cases with suspected Mendelian roots. Applying high-throughput DNA sequencing technology such as WES would shed light on new variants and delineate the genotype-phenotype correlations in clinically and genetically heterogeneous diseases such as Alazami syndrome. Further functional studies are necessary to improve our understanding of the functional impact of PTVs and the molecular mechanisms which contribute to the pathogenicity of such causative variants in LARP7. Phenotype heterogeneity will be further defined with future reports of different Alazami cases.

\section{Authors' Contribution}

$\mathrm{HN}$ is the major $\mathrm{Pl}$ of the project, the supervision, and is responsible for financial supporting, idea, design of the study, manuscript preparation, writing the original draft and review and editing. KK is responsible for clinical assessments, and examinations. GK, and FP participate in laboratory experimentation. MM, and FZA performed Sanger sequencing. SA contributed in patient recruitment. FA, and FAM had laboratory contribution. All authors agree to be accountable for all aspects of the work in ensuring that questions related to the accuracy or integrity of any part of the work are appropriately investigated and resolved.

\section{Conflict of Interest Disclosures}

The authors declare no conflict of interest.

\section{Ethical Statement}

This was a part of a grant project which was approved by the Ethics Committee of Genetics Research Center (GRC), at the University of Social Welfare and Rehabilitation Sciences (USWR), Tehran, Iran.

\section{Acknowledgements}

The authors would like to thank the patients and their families for their participation in this study. This study was funded by the Iran National Science Foundation (INSF), grant no: 96011200 (KK).

\section{References}

1. Najmabadi H, Hu H, Garshasbi M, Zemojtel T, Abedini SS, Chen W, et al. Deep sequencing reveals 50 novel genes for recessive cognitive disorders. Nature. 2011;478(7367):57-63. doi: 10.1038/nature10423.

2. Alazami AM, Al-Owain M, Alzahrani F, Shuaib $\mathrm{T}$, AlShamrani $\mathrm{H}$, Al-Falki $\mathrm{YH}$, et al. Loss of function mutation in LARP7, chaperone of 7SK ncRNA, causes a syndrome of facial dysmorphism, intellectual disability, and primordial dwarfism. Hum Mutat. 2012;33(10):1429-34. doi: 10.1002/ humu.22175.

3. He N, Jahchan NS, Hong E, Li Q, Bayfield MA, Maraia RJ, et al. A La-related protein modulates 7SK snRNP integrity to suppress P-TEFb-dependent transcriptional elongation and tumorigenesis. Mol Cell. 2008;29(5):588-99. doi: 10.1016/j. molcel.2008.01.003.

4. Hosseini M, Larti F, Fattahi Z, Najmabadi H, Kahrizi K. A splice-altering variant in LARP7 gene leads to exon exclusion. Gene Reports. 2019;15:100375. doi: 10.1016/j. genrep.2019.100375

5. Brogie JE, Price DH. Reconstitution of a functional 7SK snRNP. Nucleic Acids Res. 2017;45(11):6864-80. doi: 10.1093/nar/ gkx262.

6. Mennie AK, Moser BA, Nakamura TM. LARP7-like protein Pof8 regulates telomerase assembly and poly $(\mathrm{A})+$ TERRA expression in fission yeast. Nat Commun. 2018;9(1):586. doi: 10.1038/s41467-018-02874-0.

7. Ling TT, Sorrentino S. Compound heterozygous variants in the LARP7 gene as a cause of Alazami syndrome in a Caucasian female with significant failure to thrive, short stature, and developmental disability. Am J Med Genet A. 2016;170(1):217-9. doi: 10.1002/ajmg.a.37396.

8. Schneeberger PE, Bierhals $T$, Neu A, Hempel $M$, Kutsche $K$. de novo MEPCE nonsense variant associated with a neurodevelopmental disorder causes disintegration of 7SK snRNP and enhanced RNA polymerase II activation. Sci Rep. 2019;9(1):12516. doi: 10.1038/s41598-019-49032-0.

9. Egloff S, Vitali P, Tellier M, Raffel R, Murphy S, Kiss T. The 7SK snRNP associates with the little elongation complex to promote snRNA gene expression. EMBO J. 2017;36(7):93448. doi: 10.15252/embj.201695740.

10. Maraia RJ, Mattijssen S, Cruz-Gallardo I, Conte MR. The La and related RNA-binding proteins (LARPs): structures, functions, and evolving perspectives. WIREs RNA. 2017;8(6):e1430. doi: 10.1002/wrna.1430.

11. Dai Q, Luan G, Deng L, Lei T, Kang H, Song X, et al. Primordial dwarfism gene maintains Lin28 expression to safeguard embryonic stem cells from premature differentiation. Cell Rep. 2014;7(3):735-46. doi: 10.1016/j.celrep.2014.03.053.

12. Li H, Durbin R. Fast and accurate short read alignment with Burrows-Wheeler transform. Bioinformatics. 2009;25(14):1754-60. doi: 10.1093/bioinformatics/btp324.

13. GATK. Genome Analysis Toolkit, Variant Discovery in HighThroughput Sequencing Data. Available from: https://www. broadinstitute.org/gatk/.

14. Picard. A set of command line tools (in Java) for manipulating high-throughput sequencing (HTS) data and formats such as SAM/BAM/CRAM and VCF. Available from: http:// broadinstitute.github.io/picard/.

15. McKenna A, Hanna M, Banks E, Sivachenko A, Cibulskis K, Kernytsky A, et al. The genome analysis toolkit: A MapReduce framework for analyzing next-generation DNA sequencing data. Genome Res. 2010;20(9):1297-303. doi: 10.1101/ gr.107524.110.

16. Wang K, Li M, Hakonarson H. ANNOVAR: Functional annotation of genetic variants from high-throughput sequencing data. Nucleic Acids Res. 2010;38(16):e164. doi: 10.1093/nar/gkq603.

17. Karczewski KJ, Francioli LC, Tiao G, Cummings BB, Alföldi J, Wang Q, et al. The mutational constraint spectrum quantified from variation in 141,456 humans. Nature. 2020;581(7809):434-43. doi: 10.1038/s41586-020-2308-7.

18. Karczewski KJ, Weisburd B, Thomas B, Solomonson M, Ruderfer DM, Kavanagh D, et al. The ExAC browser: Displaying reference data information from over 60000 exomes. Nucleic Acids Res. 2017;45(D1):D840-5. doi: 10.1093/nar/gkw971.

19. Auton A, Abecasis GR, Altshuler DM, Durbin RM, Abecasis 
GR, Bentley DR, et al. A global reference for human genetic variation. Nature. 2015;526(7571):68-74. doi: 10.1038/ nature15393.

20. Glusman G, Caballero J, Mauldin DE, Hood L, Roach JC. Kaviar: An accessible system for testing SNV novelty. Bioinformatics. 2011;27(22):3216-7.doi:10.1093/bioinformatics/btr540.

21. Naslavsky MS, Yamamoto GL, de Almeida TF, Ezquina SAM, Sunaga DY, Pho N, et al. Exomic variants of an elderly cohort of Brazilians in the ABraOM database. Hum Mutat. 2017;38(7):751-63. doi: 10.1002/humu.23220.

22. Fattahi Z, Beheshtian M, Mohseni M, Poustchi H, Sellars E, Nezhadi $\mathrm{SH}$, et al. Iranome: A catalogue of genomic variations in the Iranian population. Hum Mutat. 2019;40(11):1968-84. doi: 10.1002/humu.23880.

23. Richards S, Aziz N, Bale S, Bick D, Das S, Gastier-Foster J, et al. Standards and guidelines for the interpretation of sequence variants: A joint consensus recommendation of the American College of Medical Genetics and Genomics and the Association for Molecular Pathology. Genet Med. 2015;17(5):405-24. doi: 10.1038/gim.2015.30.

24. Kopanos C, Tsiolkas V, Kouris A, Chapple CE, Albarca Aguilera $M$, Meyer R, et al. VarSome: the human genomic variant search engine. Bioinformatics. 2019;35(11):1978-80. doi: 10.1093/bioinformatics/bty897.

25. Desmet FO, Hamroun D, Lalande M, Collod-Bëroud G, Claustres M, Béroud C. Human Splicing Finder: An online bioinformatics tool to predict splicing signals. Nucleic Acids Res. 2009;37(9):e67. doi: 10.1093/nar/gkp215.

26. DTU Bioinformatics. NetGene2 Server. Available from: http:// www.cbs.dtu.dk/services/NetGene2/.

27. Hunter S, Apweiler R, Attwood TK, Bairoch A, Bateman $A$, Binns D, et al. InterPro: The integrative protein signature database. Nucleic Acids Res. 2009;37:D211-5. doi: 10.1093/ nar/gkn785.

28. Szklarczyk D, Gable AL, Lyon D, Junge A, Wyder S, HuertaCepas J, et al. STRING v11: Protein-protein association networks with increased coverage, supporting functional discovery in genome-wide experimental datasets. Nucleic Acids Res. 2019;47(D1):D607-13. doi: 10.1093/nar/gky1131.

29. Rivas MA, Pirinen M, Neville MJ, Gaulton KJ, Moutsianas L, Lindgren $\mathrm{CM}$, et al. Assessing association between protein truncating variants and quantitative traits. Bioinformatics. 2013;29(19):2419-26. doi: 10.1093/bioinformatics/btt409.

30. Liu X, Jian X, Boerwinkle E. dbNSFP v2.0: A database of human non-synonymous $\mathrm{SNVS}$ and their functional predictions and annotations. Hum Mutat. 2013;34(9):E2393-402. doi: 10.1002/humu.22376.

31. Imbert-Bouteille M, Mau Them FT, Thevenon J, Guignard T, Gatinois V, Riviere JB, et al. LARP7 variants and further delineation of the Alazami syndrome phenotypic spectrum among primordial dwarfisms: 2 sisters. Eur J Med Genet. 2019;62(3):161-6. doi: 10.1016/j.ejmg.2018.07.003.

32. Collopy LC, Ware TL, Goncalves T, Í Kongsstovu S, Yang Q, Amelina $\mathrm{H}$, et al. LARP7 family proteins have conserved function in telomerase assembly. Nat Commun. 2018;9(1):557. doi: 10.1038/s41467-017-02296-4.

33. Ivanovski I, Caraffi SG, Magnani E, Rosato S, Pollazzon M, Matalonga $L$, et al. Alazami syndrome: the first case of papillary thyroid carcinoma. J Hum Genet. 2020;65(2):13341. doi: 10.1038/s10038-019-0682-5.

34. Dateki S, Kitajima T, Kihara T, Watanabe S, Yoshiura KI, Moriuchi H. Novel compound heterozygous variants in the LARP7 gene in a patient with Alazami syndrome. Hum Genome Var. 2018;5:18014. doi: 10.1038/hgv.2018.14.

35. Hollink IHIM, Alfadhel M, Al-Wakeel AS, Ababneh F, Pfundt $R$, De Man SA, et al. Broadening the phenotypic spectrum of pathogenic LARP7 variants: Two cases with intellectual disability, variable growth retardation and distinct facial features. J Hum Genet. 2016;61(3):229-33. doi: 10.1038/ jhg.2015.134.

36. Hu H, Kahrizi K, Musante L, Fattahi Z, Herwig R, Hosseini $M$, et al. Genetics of intellectual disability in consanguineous families. Mol Psychiatry. 2018;24(7):1027-39. doi: 10.1038/ s41380-017-0012-2.

37. Wojcik MH, Linnea K, Stoler JM, Rappaport L. Updating the neurodevelopmental profile of Alazami syndrome: Illustrating the role of developmental assessment in rare genetic disorders. Am J Med Genet A. 2019;179(8):1565-9. doi: 10.1002/ ajmg.a.61189.

38. Kahrizi K, Hu H, Hosseini M, Kalscheuer VM, Fattahi Z, Beheshtian $M$, et al. Effect of inbreeding on intellectual disability revisited by trio sequencing. Clin Genet. 2019;95(1):151-9. doi: 10.1111/cge.13463.

39. Holohan B, Kim W, Lai TP, Hoshiyama H, Zhang N, Alazami $\mathrm{AM}$, et al. Impaired telomere maintenance in Alazami syndrome patients with LARP7 deficiency. BMC Genomics. 2016;17(Suppl 9):749. doi: 10.1186/s12864-016-3093-4. 